\title{
Contrôle de gestion et contingence de la performance des services publics municipaux : essai d'analyse et interprétation des pratiques
}

\author{
Donatien Avelé et Gérard Fillion \\ Université de Moncton
}

\section{INTRODUCTION}

\section{Le contrôle de gestion présente une double facette : une dimension technico- économique de nature comptable et financière complétée d'une dimension managériale et psychologique liée à la notion de contrôle et d'influence des comportements.}

Le contrôle de gestion est actuellement soumis à de nombreux questionnements. Son application dans un univers complexe, tel celui des collectivités locales, permet de faire progresser la réflexion de par son angle d'approche original et novateur. Il faut en effet attendre la décennie 1990 pour voir réellement se développer le contrôle de gestion des villes, timidement apparu entre 1977 et 1980.

Le contrôle de gestion présente une double facette: une dimension technico-économique de nature comptable et financière complétée d'une dimension managériale et psychologique liée à la notion de contrôle et d'influence des comportements ${ }^{1}$. La pratique du contrôle de gestion met effectivement en évidence l'importance de l'animation des hommes. La fonction requiert l'implication de tous et un certain degré de motivation. Elle est également censée développer l'autonomie des responsables ${ }^{2}$. Toutefois, l'emploi du terme contrôle reste ambigu. À la notion de vérification ${ }^{3}$, se substitue ou s'ajoute l'idée de planification à court terme.

La pratique du contrôle de gestion met effectivement en évidence l'importance de l'animation des hommes. La fonction requiert l'implication de tous et un certain degré de motivation.
Les organisations devenant de plus en plus complexes, il s'agit non plus de surveiller le non respect des règles et des normes mais d'instaurer la capacité de suivre les plans, et même de se les approprier. En observant cette évolution, il est possible de distinguer trois niveaux de contrôle. D'abord, le contrôle stratégique, qui consiste à définir les objectifs et les ressources nécessaires pour les atteindre. Ensuite, le contrôle de gestion permet aux managers de s'assurer que les ressources sont obtenues et utilisées de manière efficace et efficiente dans la poursuite des objectifs de l'organisation. Enfin, le contrôle opérationnel permet aux responsables de s'assurer du bon déroulement des tâches spécifiques et quotidiennes.

Le contrôle de gestion permet aux managers de s'assurer que les ressources sont obtenues et utilisées de manière efficace et efficiente dans la poursuite des objectifs de l'organisation.

L'objet de cet article est de présenter les modes et la régularité de contrôle dans les municipalités camerounaises mais aussi de fournir une perception des déterminants pouvant influencer la performance municipale. L'étude exploratoire est menée à partir des données collectées par questionnaires auprès de 50 élus locaux et de 60 chefs de services municipaux des villes camerounaises.

Dans un premier temps, nous présentons les réflexions théoriques aux modes de contrôle (1). Ensuite, nous décrivons la méthodologie de l'étude (2). Enfin, sont discutés les principaux résultats de l'étude (3). 


\section{CADRE THÉORIQUE}

L'analyse de la performance municipale suscite beaucoup d'intérêt dans les milieux académiques, mais aussi auprès des décideurs publics. La synthèse de la littérature met en relief que les déterminants de la performance sont nombreux et variés. Sur ce point, le modèle conceptuel, proposé dans le cadre de cette étude, retient un certain nombre de variables, tel qu'illustré à la figure 1 .

Figure 1 : Cadre conceptuel des déterminants de la performance municipale

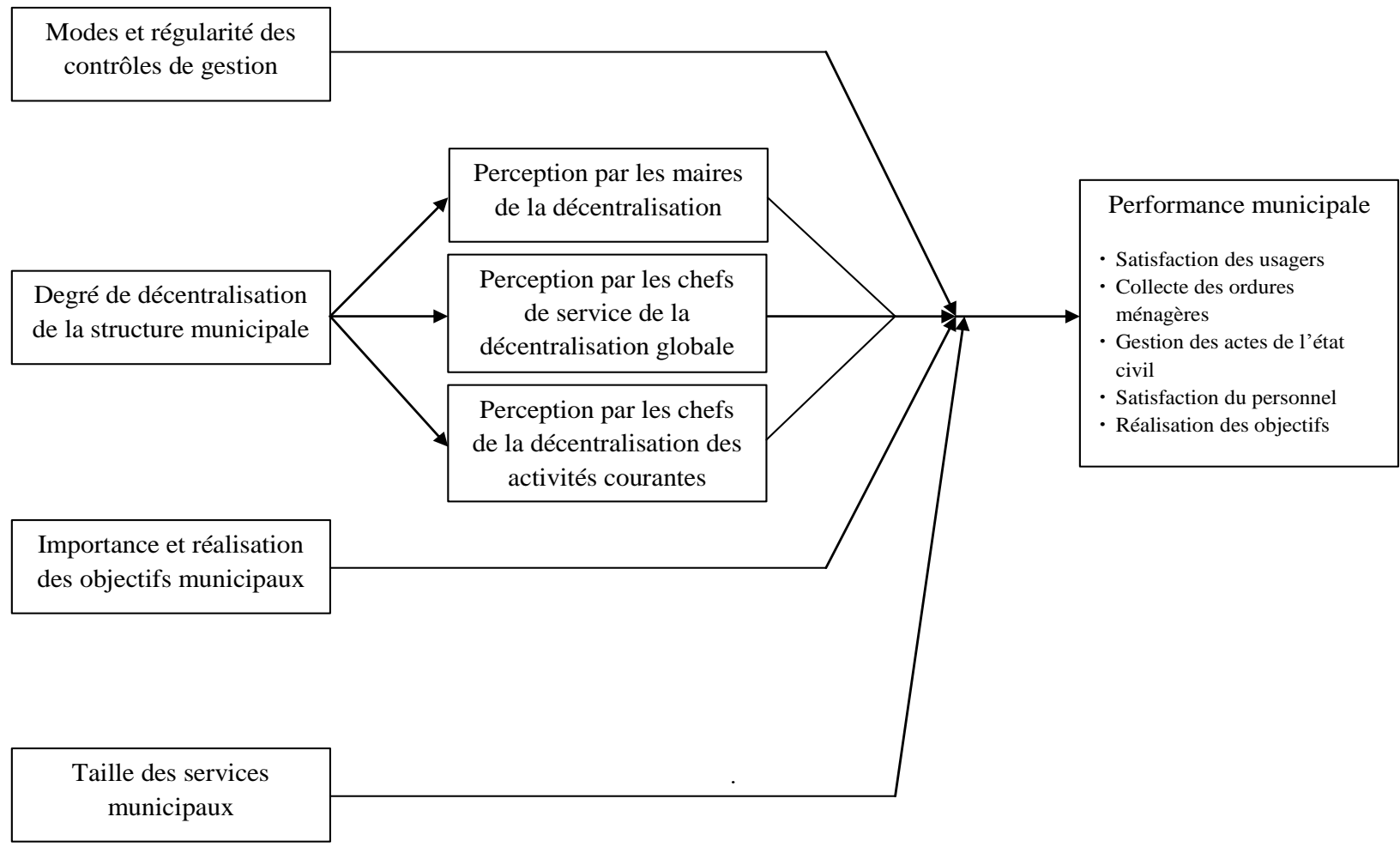

La décentralisation doit s'accompagner d'une forte structuration des activités, c'est-à-dire une définition précise des tâches et des rôles de chacun pour maintenir le contrôle. Dans une organisation décentralisée et structurée, les activités sont clairement définies, les aires de responsabilité et d'autorité sont clairement délimitées, et le contrôle est essentiellement impersonnel. Les décisions peuvent donc être déléguées puisque le contrôle organisationnel est maintenu. La décentralisation fait référence au degré d'autonomie laissé aux responsables ${ }^{5}$. La liberté d'action des divers responsables de l'organisation a été définie à différents niveaux hiérarchiques pour un certain nombre de décisions. En référence aux travaux de recherche ${ }^{6}$, il se dégage clairement que la structure organisationnelle a fait l'objet de nombreuses études empiriques dans les organisations. De ce fait, il serait intéressant de voir si la structure municipale peut influencer la performance des services publics municipaux des villes camerounaises. Il sera donc question, dans le cadre de cette étude, de tester l'hypothèse à l'effet qu'il existe une relation positive entre le degré de décentralisation de la structure municipale et la performance des services publics municipaux des villes camerounaises.

Par ailleurs, d'autres travaux ont mis en évidence l'influence de la taille de l'organisation sur la performance. Les principaux résultats de ces travaux confortent l'idée selon laquelle la taille de l'organisation constitue un facteur pré- 
dictif majeur de sa structuration. Pour ce qui est de la relation taille/structure, de nombreux travaux empiriques confirment cette proposition ${ }^{7}$. Toutefois, une étude sur l'usage des tableaux de bord dans 82 municipalités françaises montre qu'une large majorité des villes n'utilise pas de tableaux de bord pour contrôler le fonctionnement des services et piloter l'activité municipale $^{8}$. Selon cette étude, «on ne peut donc affirmer, avec certitude, que taille et utilisation des tableaux de bord sont corrélées, d'autant que la nature de ces tableaux de bord peut être varia-

\section{MÉTHODOLOGIE DE L'ÉTUDE}

L'enquête a été réalisée auprès des communes $\mathrm{du}$ Cameroun. Les communes camerounaises représentaient un ensemble de collectivités réparties en 2 communautés urbaines, 11 communes urbaines d'arrondissement subdivisant les deux communautés urbaines de Douala et de ble ». Au regard de ce qui précède, la taille de l'organisation apparaît donc comme une variable susceptible d'influer sur les caractéristiques de la performance des services publics municipaux des villes camerounaises. En nous replaçant dans le contexte de l'étude et, s'inspirant des travaux antérieurs, il serait opportun d'examiner si la taille élevée des services municipaux entraîne une meilleure performance. Il serait aussi pertinent de voir si la taille élevée est corrélée avec une utilisation accrue des tableaux de bord.

Yaoundé dont 5 à Douala et 6 à Yaoundé, 20 communes urbaines dont 11 à régime spécial et 306 communes rurales. Le tableau 1 résume les différents types de communes et de services ayant participé à l'enquête.

Tableau 1 : Types de communes et de services à l'enquête quantitative

\begin{tabular}{|l|l|l|c|}
\hline \multicolumn{2}{|l|}{ Types de communes } & \multicolumn{2}{|l|}{ Types de services (148) } \\
\hline Communes rurales & $84(82 \%)$ & Services financiers & $56(38 \%)$ \\
\hline Communes urbaines & $05(5 \%)$ & Services techniques & $40(27 \%)$ \\
\hline $\begin{array}{l}\text { Communes urbaines } \\
\text { d'arrondissement }\end{array}$ & $11(10,78 \%)$ & $\begin{array}{l}\text { Services } \\
\text { administratifs }\end{array}$ & $52(35 \%)$ \\
\hline Communes urbaines à régime spécial & $02(1,96 \%)$ & \multicolumn{1}{c|}{ Totaux } & - \\
\hline Totaux & $\mathbf{1 0 2 ( 1 0 0 \% )}$ & \multicolumn{1}{|c|}{} \\
\hline
\end{tabular}

Pour répondre à la problématique posée et tester ainsi les hypothèses formulées dans la recherche, nous avons décidé de retenir le questionnaire comme mode principal de recueil de données. Au total, 250 questionnaires ont été administrés à la fois aux élus et aux responsables de diffé- rents services publics municipaux. Sur les 250 questionnaires administrés, 102 ont été adressés aux élus et 148 aux responsables de services. Les détails des taux de réponse relatifs aux questionnaires retournés, exploitables et non exploitables sont fournis dans le tableau 2.

Tableau 2 : Taux de réponse à l'enquête quantitative

\begin{tabular}{|l|l|c|l|}
\hline & Elus & Chefs de services & Totaux \\
\hline Questionnaires envoyés & $102(100 \%)$ & $148(100 \%)$ & $250(100 \%)$ \\
\hline Questionnaires retournés & $62(60,78 \%)$ & $88(59,45 \%)$ & $150(60 \%)$ \\
\hline Questionnaires non exploitables & $12(11,76 \%)$ & $28(18,91 \%)$ & $40(16 \%)$ \\
\hline Questionnaires exploitables & $\mathbf{5 0 ( 4 9 \% )}$ & $\mathbf{6 0 ( 4 0 , 5 4 \% )}$ & $\mathbf{1 1 0}(\mathbf{4 4 \%})$ \\
\hline
\end{tabular}


Le taux de réponse de l'ensemble des questionnaires exploitables est de $44 \%$. Nous l'avons jugé satisfaisant compte tenu du caractère exploratoire de la recherche et surtout de l'hostilité dont font face les acteurs municipaux dans les pays en voie de développement à l'instar du

\section{PRINCIPAUX RÉSULTATS ET DISCUSSION}

\subsection{Modes et régularité des contrôles dans les 50 municipalités de l'échantillon}

Le contrôle de légalité est un contrôle exercé par les représentants de l'État auprès des municipalités pour faire respecter la légalité et préserver l'intérêt de l'État tout en évitant les abus de tout genre.

Les résultats de l'enquête quantitative démontrent qu'il existe trois types de contrôles dans les 50 mairies constituant l'échantillon de notre étude. D'abord, le contrôle de légalité est un contrôle exercé par les représentants de l'État auprès des municipalités pour faire respecter la légalité et préserver l'intérêt de l'État tout en évitant les abus de tout genre. Ensuite, le contrôle d'exécution est assimilé à la hiérarchie, car il permet aux responsables municipaux (magistrats municipaux, cadres, et agents territoriaux) de s'assurer que les actions qui relèvent de leur autorité sont mises en œuvre conformément aux objectifs, tout en dispensant ces responsables de piloter eux-mêmes ces actions ${ }^{9}$.
Cameroun. Compte tenu de la taille limitée de notre échantillon, mais aussi des objectifs de l'étude, nous avons opté pour des tests non paramétriques en utilisant le logiciel Statistical Package for the Social Sciences (SPSS) pour le traitement des données de l'étude.

Il est effectué par les représentants du ministère de l'économie et des finances en l'occurrence les contrôleurs financiers. Enfin, le contrôle de gestion occupe une place essentielle, puisqu'il est le pivot entre la politique générale de l'organisation et les tâches quotidiennes des opérationnels ${ }^{10}$. Comme l'illustre bien le graphique 1 , les résultats de l'enquête quantitative montrent que seulement $10 \%$ des 50 mairies de l'échantillon possèdent un service de contrôle de gestion contre $90 \%$ qui ne le possèdent pas. Les contrôles légal et financier exercés par les inspecteurs issus des ministères de tutelle sont généralisés dans l'ensemble des mairies camerounaises visitées.

Les contrôles légal et financier exercés par les inspecteurs issus des ministères de tutelle sont généralisés dans l'ensemble des mairies camerounaises visitées.

\section{Graphique 1 : Existence du contrôle de gestion dans les 50 mairies}

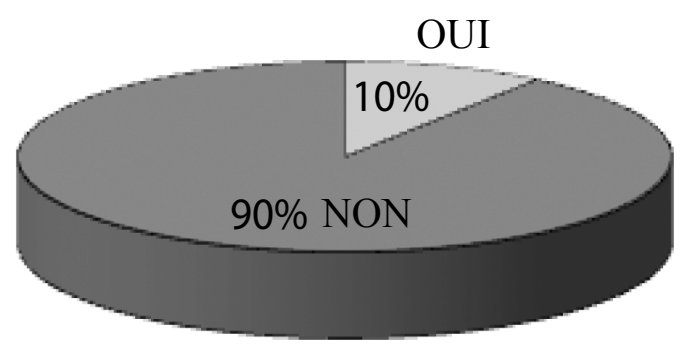


Par ailleurs, la régularité des contrôles concerne les contrôles exercés par la tutelle. Les résultats de l'enquête quantitative montrent que $36 \%$ des mairies de l'échantillon sont inspectées par les représentants de la tutelle dans une fréquence variant de 4 à 6 fois au cours d'un même exercice budgétaire. En revanche, $28 \%$ des mairies reçoivent moins de trois contrôles et $8 \%$ seulement des mairies reçoivent plus de dix contrôles par an. Le graphique 2 fournit plus de détails sur la régularité des contrôles exercés par la tutelle au sein des mairies camerounaises ou tout au moins sur les 50 mairies de l'échantillon.

\section{Graphique 2 : Régularité des contrôles dans les 50 mairies de l'échantillon}

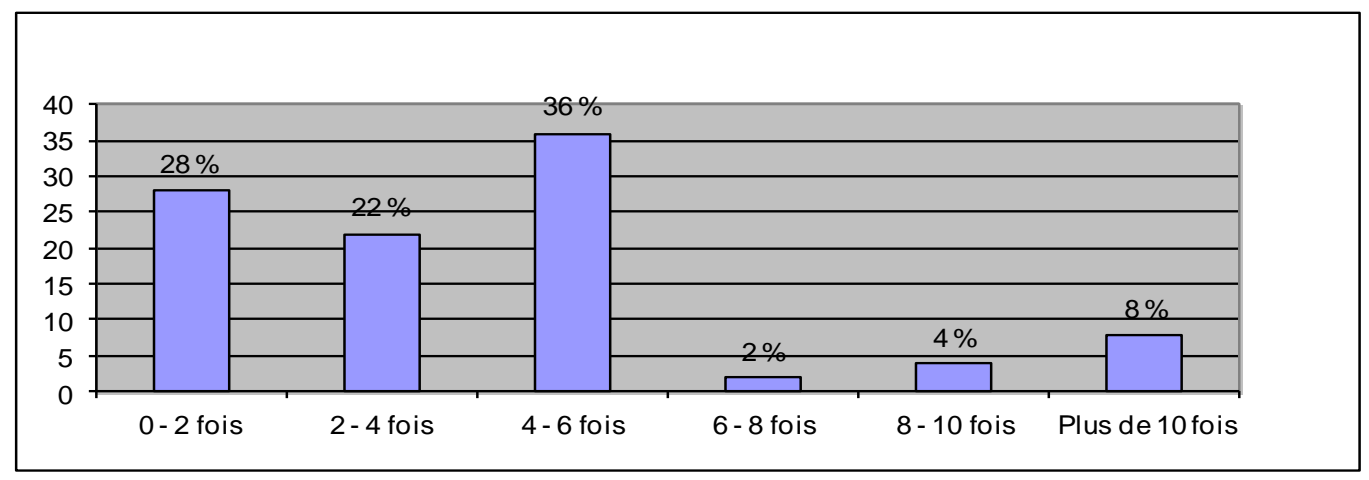

3.2 Une comparaison de la perception de certains facteurs pouvant influencer la performance municipale par les maires et chefs de services

Nous retenons les 50 mairies et 60 services municipaux de l'échantillon pour mesurer le degré de décentralisation des services municipaux. Nous ne considérons pas la décentralisation du seul point de vue de l'autonomie juridique, mais aussi de l'autonomie réelle du service, c'est-àdire la liberté d'action au jour le jour.

\subsubsection{La perception par les maires de la décentralisation}

La question posée pour mesurer le degré de décentralisation des services municipaux était construite sur une échelle à cinq points allant de «pas du tout d'accord» à «tout à fait d'accord ». Les répondants devaient nous indiquer si la structure municipale de leur mairie était totalement décentralisée. Les résultats de l'enquête quantitative sur cette dimension sont présentés au graphique 3. Ils mettent en relief l'existence d'une note extrême car 27 maires soit $54 \%$ ne sont pas du tout d'accord de l'opinion selon laquelle leur structure municipale est totalement décentralisée. Nous avons donc là une répartition disparate entre les 50 maires de l'échantillon. $26 \%$ sont peu d'accord à ce que leur mairie soit totalement décentralisée, $18 \%$ sont moyennement d'accord contre $2 \%$ seulement qui sont d'accord. En revanche, aucun des 50 maires n'est tout à fait d'accord quant à la décentralisation totale de sa mairie.

Graphique 3 - Le degré de décentralisation perçu par les 50 maires de l'échantillon

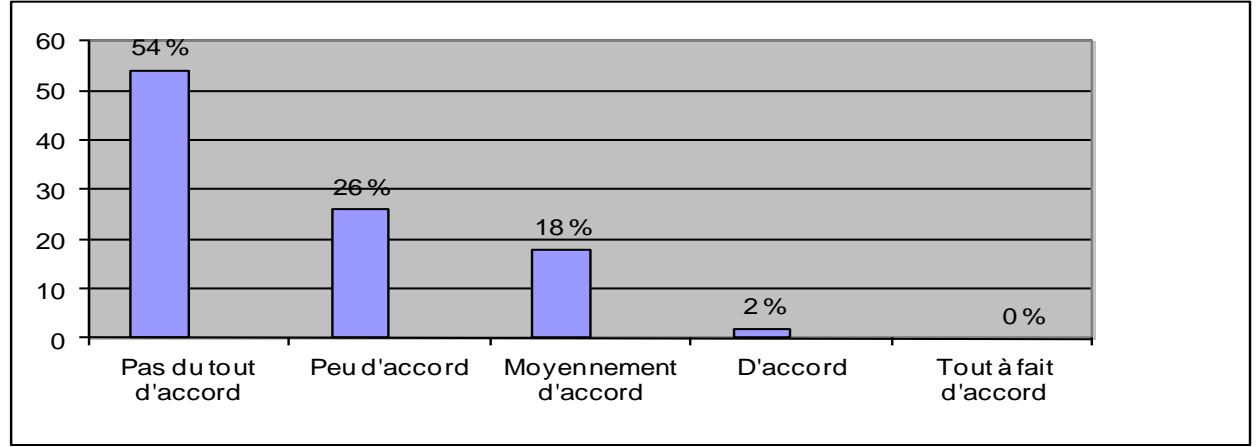


Ces résultats rejoignent certains travaux qui montraient une répartition inégale des opinions des chefs de services par rapport à la décentralisation de certains actes ${ }^{10}$. Il existe en effet une importante dispersion des avis des répondants sur le degré de décentralisation des services publics municipaux des villes camerounaises.

\subsubsection{La perception par les chefs de service de la décentralisation globale}

\section{La perception des maires et de leurs chefs de service sur la décentralisation n'est pas uniforme et identique. Le score moyen est plus élevé chez les chefs de service que chez les maires.}

Afin de mesurer cette autonomie, nous avons demandé aux responsables des services publics municipaux s'ils appartenaient à une mairie globalement décentralisée. Nous mesurons ici une perception de l'autonomie. Mais cette " impression » est essentielle dans la mesure où elle est celle des chefs de service qui sont responsables de la mise en place d'outils de gestion nécessaires au contrôle de la performance des services publics municipaux des villes camerounaises. Tout comme les maires, les personnes interrogées, en l'occurrence les chefs de service, devaient répondre sur une échelle en cinq points allant de " pas du tout d'accord» à «tout à fait d'accord». Les résultats obtenus lors de l'enquête de terrain se répartissent dans le graphique 4.

\section{Graphique 4 : La décentralisation perçue par les 60 chefs de service de l'échantillon}

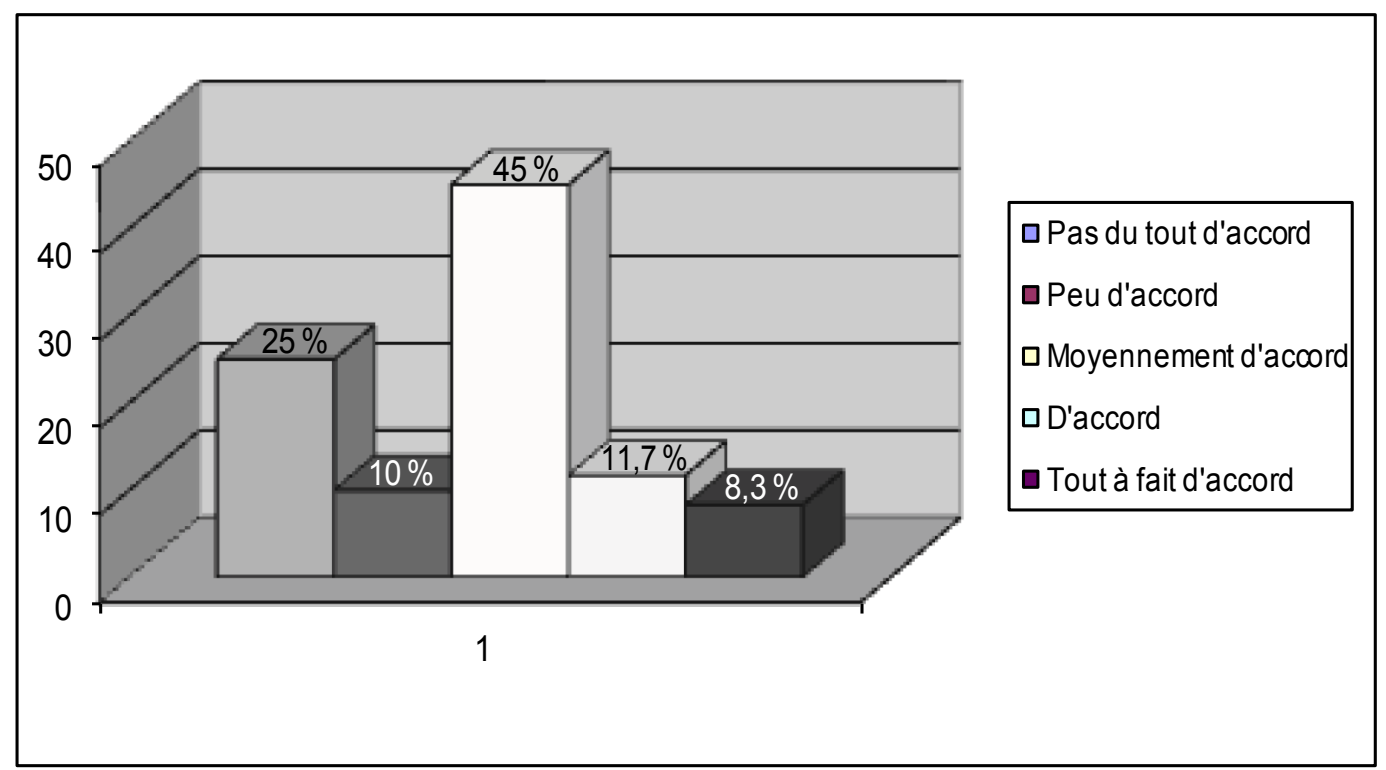

Les résultats montrent que $25 \%$ des chefs de service interrogés ne sont pas du tout d'accord quant à leur appartenance à une mairie totalement décentralisée. Toujours dans la même logique, $10 \%$ des chefs de services sont peu d'accord contre $45 \%$ qui sont moyennement d'accord. De l'autre côté, $11,7 \%$ des chefs de service seulement sont d'accord quant à la décentralisation totale de leur mairie par rapport à l'appartenance de leur service et $8,3 \%$ sont tout à fait d'accord. En définitive, la perception des maires et de leurs chefs de service sur la décentralisation n'est pas uniforme et identique. Le score moyen est plus élevé chez les chefs de service que chez les maires. Il existe donc une forte dispersion des scores entre les personnes ayant pris part à l'enquête. Cette question ne faisant apparaître qu'une perception très globale de l'autonomie, il nous a semblé indispensable d'évaluer un peu plus objectivement la marge de manœuvre de chacun à la tête de son service. Nous avons donc interrogé chaque responsable sur son degré d'autonomie pour quelques décisions importantes. 


\subsubsection{La perception par les chefs de service de la décentralisation de certains actes courants}

En dehors des gros investissements qui sont du ressort des décisions politiques et donc des maires, les résultats de l'étude montrent que les élus des 50 mairies laissent une bonne marge de manœuvre à leurs responsables des différents services municipaux pour la gestion courante des activités quotidiennes.
Nous tentons d'appréhender la décentralisation de certains actes à partir de sept types de décisions fondamentales pour le bon fonctionnement d'un service municipal soit les achats de petits matériels, les achats de consommables, l'augmentation de crédits, la décision budgétaire, le recrutement des agents, la définition des méthodes de travail et la répartition des tâches. Le graphique 5 présente la perception des chefs de services.

\section{Graphique 5 : La perception par les 60 chefs de service de l'autonomie de certains actes}

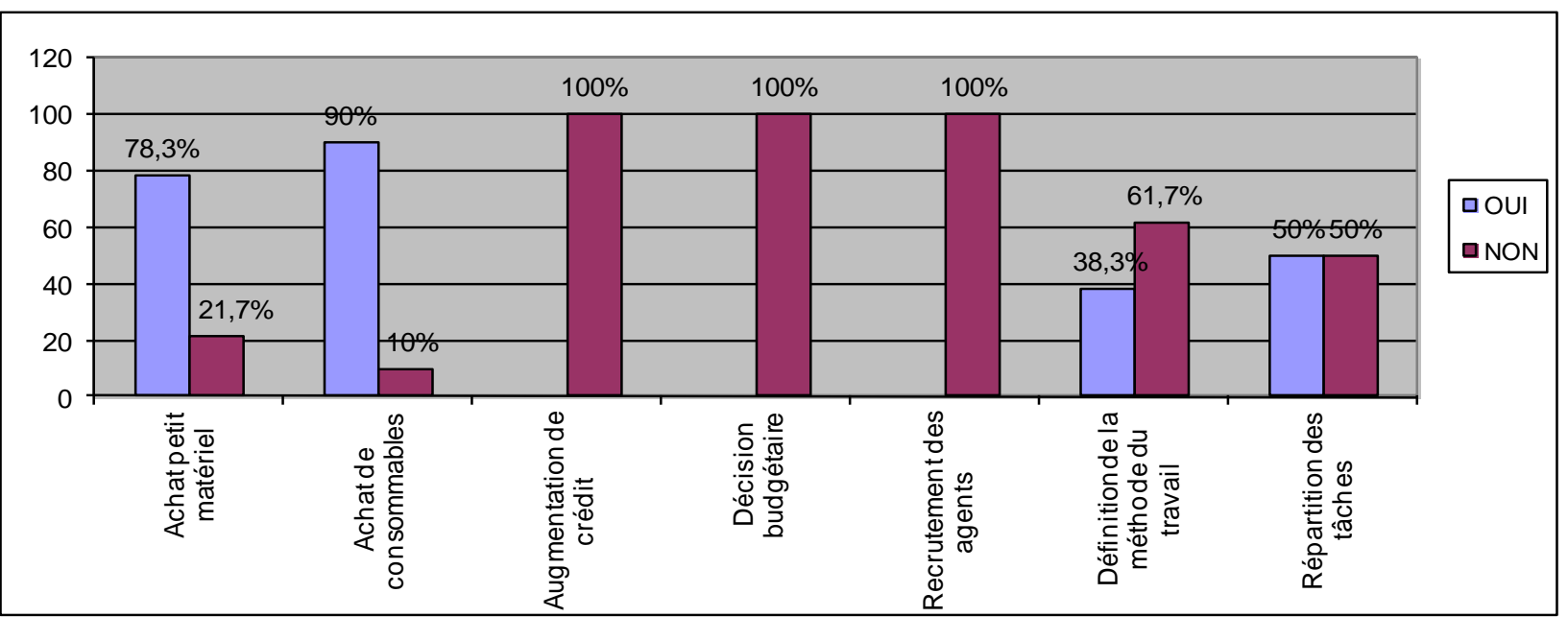

Pour chacun des types d'activités, chaque chef de service indique en cochant la case appropriée les actes pour lesquels il était autonome. Les résultats de l'enquête quantitative montrent qu'une large majorité des chefs de service des municipalités visitées détiennent sans doute une véritable autonomie quant aux achats des petits matériels et consommables; soit respectivement $78,3 \%$ et $90 \%$ contre seulement $21,7 \%$ qui ne détiennent pas cette autonomie pour les achats des petits matériels et $10 \%$ pour les consommables. Nous avons observé que les achats du petit matériel ou des consommables constituent bien un acte majeur pour le bon fonctionnement d'une activité dans un service. En dehors des gros investissements qui sont du ressort des décisions politiques et donc des maires, les résultats de l'étude montrent que les élus des 50 mairies laissent une bonne marge de manœuvre à leurs responsables des différents services municipaux pour la gestion courante des activités quotidiennes.
S'agissant des augmentations de crédits, des décisions budgétaires ou encore du recrutement des agents, les résultats de l'enquête de terrain montrent bien que la liberté de l'action des chefs de service est réduite à néant. Les 60 chefs de service de l'échantillon ont répondu ne pas détenir la moindre liberté quant à ces différents actes qui dans l'ensemble relèvent de la compétence politique et donc des élus et surtout lors des votes budgétaires. Sur le plan du recrutement des agents, les possibilités d'intervention des chefs de services sont en fait peu nombreuses, voire inexistantes au regard des résultats de l'enquête. Les chefs de service ne participent nullement aux commissions de recrutement pour juger des qualités techniques des candidats. Le recrutement relève de la seule compétence du maire. Il est le seul à juger des compétences et des qualités techniques des agents qu'il souhaite recruter pour répondre aux besoins des services. Même les secrétaires généraux ne sont pas consultés. Les agents sont purement et simplement imposés aux chefs de service, soit par 
les élus soit par les recommandations émanant le plus souvent des hommes politiques ou encore de la tutelle. Pour ce qui est de la définition des méthodes de travail et de la répartition des tâches, $61,7 \%$ des responsables de service sont autonomes pour l'acte de la définition des méthodes de travail et $50 \%$ pour celui concernant la répartition des tâches. Nous observons là une concentration des pouvoirs encore entre les mains du «super chef » qu'est le maire pour certains services.

$\mathrm{Au}$ final, la décentralisation est d'autant moins effective que les décisions auxquelles les responsables de services contribuent sont soumises à des contraintes légales fortes (cas des décisions budgétaires ou encore des augmentations de crédits) ou qu'elles ont des conséquences sur les services à moyen et long terme (cas du recrutement du personnel).

\subsection{Le degré d'importance et la réalisation des objectifs municipaux par les 50 maires de l'échantillon}

La définition précise des objectifs organisationnels est indispensable pour répondre aux besoins des populations. La mesure de la performance des services publics municipaux permet ainsi d'apprécier l'atteinte des objectifs. Elle est un moyen au service du secrétariat général pour suivre l'exécution des décisions et assurer la cohérence entre la stratégie et l'action quotidienne. Nous mesurons aussi le degré d'importance de la satisfaction des usagers consommateurs de la collecte des ordures ménagères, de la gestion des actes de l'état civil, de la satisfaction du personnel et de l'approvisionnement en eau potable. Pour chacune de ces dimensions, les personnes répondantes devaient répondre sur une échelle en cinq points allant de «très important » à «pas du tout important». Les résultats sont fournis dans le graphique 6.

\section{Graphique 6 : Le degré d'importance des objectifs municipaux}

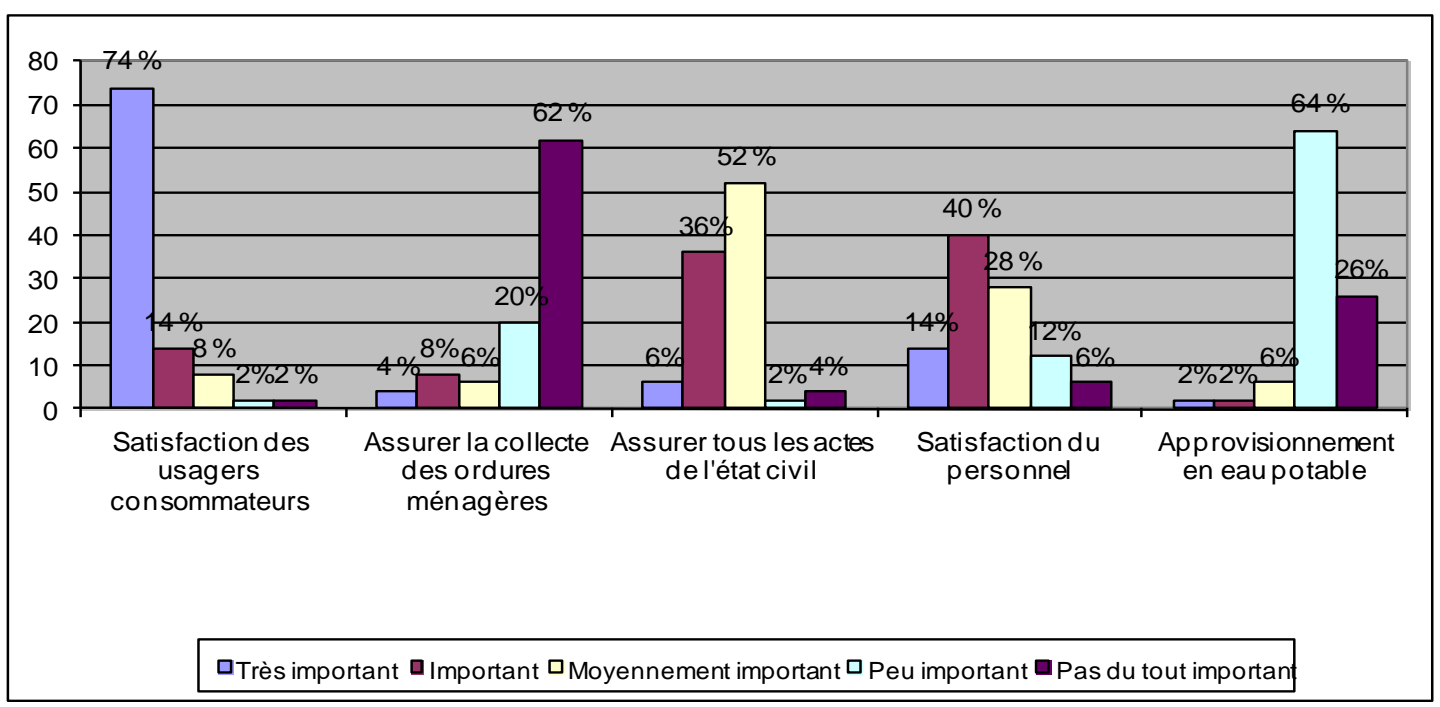

Les résultats de l'enquête quantitative montrent que $74 \%$ des répondants considérèrent que l'objectif d'assurer la satisfaction des usagers est très important. S'agissant de l'importance de l'objectif d'assurer la collecte des ordures ménagères, $4 \%$ seulement des répondants considèrent que celui-ci est très important, $8 \%$ le considèrent comme étant important, $6 \%$ moyennement important, $20 \%$ peu important. $36 \%$ des personnes interrogées pensent que la gestion des actes de l'état civil est importante alors que $52 \%$ les trouvent moyennement importants. Enfin, le nombre de personnes interrogées qui pensent que l'objectif d'approvisionnement en eau potable est très important semble globalement très faible. La raison que l'on peut donner à cet état de chose est le fait que l'approvisionnement en eau potable est une activité très coûteuse pour les mairies camerounaises. Elles se trouvent ainsi dans l'obligation de partager la gestion de cette activité avec une entreprise privée qu'est la société nationale des eaux du Cameroun. 


\subsection{Influence des facteurs contingents sur la performance des services publics municipaux}

\section{L'analyse des relations entre la décentralisation de la mairie et la réalisation des objectifs fait apparaître un lien significatif. Les mairies les plus décentralisées sont celles qui réalisent au mieux la performance municipale.}

L'analyse empirique met en relief l'existence de corrélations positives entre les variables de contingence structurelles et la performance des services publics municipaux. Selon les résultats des tests statistiques, il existe une relation positive entre le degré de décentralisation de la structure municipale et la performance des services publics municipaux des villes camerounaises. L'analyse des relations entre la décentralisation de la mairie et la réalisation des objectifs fait apparaître un lien significatif. Les mairies les plus décentralisées sont celles qui réalisent au mieux la performance municipale. En d'autres termes, cela indique que plus la municipalité est décentralisée, plus les services sont tout aussi décentralisés et plus les objectifs sont aisément atteints. Nous avons observé que les mairies qui centralisent certaines décisions (par exemple l'achat des petits matériels nécessaires au fonctionnement du service) rendent plutôt le fonctionnement de ces services lourds. Par ailleurs, les résultats de l'enquête quantitative montraient déjà qu'une large majorité des chefs de service des municipalités visitées détiennent sans doute une véritable autonomie quant aux achats des petits

\section{CONCLUSION}

Cet article a rendu compte des résultats d'une étude empirique menée dans les municipalités des villes camerounaises. Il a mis en lumière les modes et la régularité des contrôles existants dans ces municipalités. Il a tout aussi montré que les contrôles de gestion sont pour la plupart exercés par les ministères de tutelles des municipalités camerounaises. Selon l'enquête sur la perception des maires et des chefs de service des municipalités étudiées, il est démontré que $74 \%$ des personnes répondantes (maires et chefs de service) estiment que l'objectif le plus important est la satisfaction des usagers consommateurs. Il ressort aussi que le recrutement ne relève que de la seule compétence du maire. Il est le seul à juger matériels et consommables. Cela témoigne bien l'influence que peut avoir la décentralisation sur la performance des services publics municipaux.

L'analyse empirique porte aussi à croire que la taille élevée des services municipaux n'incite pas les responsables des services à faire usage des tableaux de bord. Le coefficient de corrélation ne révèle aucun lien significatif entre la taille des services et l'usage des tableaux de bord. Les résultats indiquent en revanche qu'il existe une relation significative entre l'utilisation des tableaux de bord et la réalisation des objectifs. En d'autres termes, plus on utilise les tableaux de bord dans les services plus on atteint les objectifs de performance municipale.

Enfin, l'analyse empirique démontre que plus les responsables des services municipaux ont recours à l'informatisation des activités, plus ils améliorent la performance municipale. La corrélation positive au terme du croisement des deux variables indique que les municipalités possédant un nombre important d'ordinateurs réalisent souvent leurs objectifs.

Les résultats indiquent en revanche qu'il existe une relation significative entre l'utilisation des tableaux de bord et la réalisation des objectifs. En d'autres termes, plus on utilise les tableaux de bord dans les services plus on atteint les objectifs de performance municipale.

des qualités techniques et des compétences des agents que la municipalité doit embaucher. L'action des chefs de service s'avère également limitée en ce qui concerne les décisions relatives à l'augmentation des crédits.

Selon les résultats de cette étude, la décentralisation de la structure municipale améliore la performance des services municipaux. Il ressort cependant que la taille des services municipaux n'a pas d'influence sur le degré d'utilisation des tableaux de bord. De plus, il y a une relation significative entre l'utilisation des tableaux de bord et la réalisation des objectifs municipaux et donc de la performance municipale. 
Une volonté accrue de la part des acteurs municipaux des villes camerounaises par la mise en place d'un véritable système de contrôle de gestion régulière et d'une véritable gestion des ressources humaines permettrait d'améliorer les performances municipales. De plus, la mise en place par exemple d'une politique de benchmarking pourrait être une très bonne initiative dans un contexte de coopération nord-sud. Un tel dispositif viendra en complément des relations déjà existantes (Bordeaux et Douala) ou encore (Québec-ville et Douala) et serait favorable à des pistes d'amélioration de la performance municipale au Cameroun.

\section{BIBLIOGRAPHIE}

${ }^{1}$ Naro G. (1995). «Les apports de la GRH au contrôle de gestion : une revue de la littérature anglo-saxonne», Colloque de Toulouse: Comptabilité et GRH, 12 juin, AFC et AFGRH, 52-66.

${ }^{2}$ Hofstede G. (1981). "Management Control of Public and not-for-profit Activities" Accounting, Organizations and Society, vol. 6, $\mathrm{n}^{\circ}$ 3, 1993-211.

${ }^{3}$ Fayol H. (1916). Administration Industrielle et Générale, Paris.

${ }^{4}$ Anthony R.N. (1965). Planning and Control System, a Framework for Analysis, Division or Research, Harvard Business School, $180 \mathrm{p}$.

${ }^{5}$ Chenhall, R et Morris, D. (1986). «The Impact of Structure, Environment and Interdependence on the Perceived Usefulness of Management Accounting Systems». Accounting Review, 61, 16-35.

${ }^{6}$ Bruns, W.J. et Waterhouse, J.H. (1975). « Budgetary Control and Organization Structure», Journal of Accounting Research, 177-205.

${ }^{7}$ Desreumaux A. (1992). Structures d'entreprises, Analyse et Gestion, Vuibert; Pugh D.S. et al. (1969), «An Empirical Taxonomy of Structures of Work Organisations», Administrative Science Quarterly, September, 115-126; Blau, P.M. et Schoennherr, R.A. (1971). The Structure of Organization, Basic Books, NewYork.

${ }^{8}$ Meyssonier F. (1993). Le contrôle de gestion communal : bilan et perspectives, Thèse de doctorat en sciences de gestion, Université Nancy II.

${ }^{9}$ Burlaud A. et Malo J.L. (1988). «Les organisations complexes: un défi aux méthodes traditionnelles de contrôle de gestion » Revue Française de Gestion, Février, 58-64. Khandwalla P.N. (1972). «The Effect of Different Types of Competition on the Use of Management Controls », Journal of Accounting Research, 275-285.

${ }^{10}$ Roussarie O. (1994). Les outils de contrôle de gestion utilisés dans les services publics urbains : observation et interprétation des pratiques, Thèse de doctorat en Sciences de gestion, Université de Poitiers. 